\title{
Morphological Study of Ceramic Hollow Fibre Membranes
}

\author{
Benjamin F. K. Kingsbury and K. Li* \\ Department of Chemical Engineering, Imperial College London, South Kensington Campus, London \\ SW7 2AZ, UK.
}

\begin{abstract}
Morphologies of ceramic hollow fibre membranes prepared from suspensions of $\mathrm{Al}_{2} \mathrm{O}_{3}$, NMP (Nmethyl-2-pyrrolidone) and polyethersulfone (PESf) using a dry-wet spinning/sintering process have been studied experimentally. The results indicate that two types of membrane morphologies, i.e. fingerlike and sponge-like structures can be expected. It is believed that finger-like void formation in asymmetric ceramic membranes is initiated by hydrodynamically unstable viscous fingering developed when a less viscous fluid (non-solvent) is in contact with a higher viscosity fluid (ceramic suspension containing invertible polymer binder). Finger-like void growth occurs only below a critical suspension viscosity, above which a sponge-like structure is observed over the entire hollow fibre cross section. The effects of the air-gap, viscosity and non-solvent concentration on fibre morphology have been studied and it has been determined that viscosity is the dominating factor for ceramic systems.
\end{abstract}

Keywords: Alumina, Asymmetric Membrane, Hollow fibres, Morphology

*To whom correspondence should be addressed

Telephone: 44 (0) 207-5945676

Fax: 44 (0) 207-5945629

Email: Kang.Li@imperial.ac.uk

Revised manuscript submitted to Journal of Membrane Science 21 October 2008 


\section{Introduction}

The combination of high chemical, thermal and mechanical resistance has made ceramic membranes an attractive alternative to polymeric varieties [1,2]. Due to the high surface area/volume ratios achieved by hollow fibre configurations, ceramic hollow fibre membrane performance may greatly exceed that of other membrane systems. The ability to operate at high temperatures and pressures, and in corrosive environments, allows ceramic membranes to be used in a variety of applications including filtration for corrosive fluids [3], high temperature membrane reactors [4-6], solid oxide fuel cells [7] and membrane contactors [8] as well as robust membrane supports [9]. In particular, the solvent resistant nature of ceramic membrane materials makes them extremely useful for solvent filtration purposes as they can be used with a variety of both polar and apolar solvents.

Production of ceramic hollow fibre membranes can be achieved using an immersion induced phase inversion method first described by Loeb and Sourirajan [10] for asymmetric polymeric membrane formation. After the addition of a desired amount of ceramic powder to a polymer solution, if well mixed, the ceramic/polymer/solvent system can be seen as a suspension of polymer coated ceramic particles. Once immersed in a non-solvent for the polymer which is miscible with the solvent, solvent/non-solvent exchange takes place leading to precipitation of the polymer phase. Ceramic particles are immobilized once precipitation has taken place and the membrane macrostructure can be largely determined at this point by manipulating and adjusting the various parameters of the phase inversion process.

Great effort has been made to both control and understand the formation mechanisms for the wide range of structures observed in polymeric membrane formation [11-17]. However, due to the large differences between polymeric and ceramic systems, in particular the low polymer concentration, this information is of limited use during ceramic membrane preparation. In fact, only two morphologies have so far been observed in ceramic/polymer systems, i.e. (1) finger-like voids and (2) a sponge-like structure. The macrostructure of the fibre precursor designed during the phase inversion process is retained during sintering (calcination/heat treatment). Finger-like voids above a certain size are not usually eliminated, although at elevated sintering temperature sponge-like regions will densify and eventually become gas tight for some ceramic materials [18]. The present work is, therefore, to examine how the choice of process parameters affects ceramic membrane morphology.

Hydrodynamically unstable viscous fingering is a well known phenomenon that occurs at the interface between fluids with different viscosities in the first moments of mixing and has been applied here to 
explain the formation of finger-like voids in ceramic membrane precursors. When the suspension is in contact with non-solvent, a steep concentration gradient results in solvent/non-solvent exchange, a rapid increase in local viscosity and finally precipitation of the polymer phase. However, due to instabilities at the interface between the suspension and the coagulant there is a tendency for viscous fingering to occur, initiating the formation of finger-like voids. The relative thickness of finger-like and sponge-like regions greatly affects the properties of the membrane such as membrane mechanical strength and permeation flux and, due to the versatility of ceramic hollow fibre membranes, it is essential that fibre morphology can be controlled so that it may be tailored to a specific application.

In this study, it has been shown that finger-like voids can be initiated from both the inner and outer surfaces simultaneously or from the inner surface alone. Finger-like void length from the inner surface can be varied between $0 \%$ of the fibre thickness, i.e. a symmetric sponge-like membrane structure, to approximately $80 \%$ or above of the fibre cross-section. The density of the outer sponge-like region can also be controlled. A hypothesis for the prediction of the length of finger-like voids, the thickness of the sponge-like region and the formation and elimination of isolated/encapsulated voids has been suggested and correlated with air-gap, suspension viscosity, non-solvent additive and internal coagulant flow rate.

\section{Experimental}

\section{Materials}

Aluminium oxide powders $1 \mu \mathrm{m}$ (alpha, $99.9 \%$ metals basis, surface area 6-8 m²/g), $0.3 \mu \mathrm{m}$ (gammaalpha, 99.9 \% metals basis, surface area $15 \mathrm{~m}^{2} / \mathrm{g}$ ), $0.05 \mu \mathrm{m}$ (gamma-alpha, $99.5 \%$ metals basis, surface area 32-40 $\mathrm{m}^{2} / \mathrm{g}$ ) and $0.01 \mu \mathrm{m}$ (gamma-alpha, $99.98 \%$ metals basis, surface area $100 \mathrm{~m}^{2} / \mathrm{g}$ ) were purchased from Alfa Aesar (a Johnson Matthey company) and were used as supplied. Polyethersulfone (Radal A300, Ameco Performance, USA), N-methyl-2-pyrrolidone (HPLC grade, Rathbone) and Arlacel P135 (Polyethyleneglycol 30-dipolyhydroxystearate, Uniqema) were used as binder, solvent and additive, respectively. Tap water and de-ionized water were used as the external and internal coagulants, respectively.

\section{Preparation of alumina hollow fibres}


Arlacel P135 at a concentration of $1.3 \mathrm{wt} \%$ was dissolved in NMP/water solutions prior to the addition of aluminum oxide powders (58.7 wt\%) at a ratio of 1:2:7 (0.01 $\mu \mathrm{m}: 0.05 \mu \mathrm{m}: 1 \mu \mathrm{m})$. The dispersion was rolled/milled with $20 \mathrm{~mm}$ agate milling balls with an approximate $\mathrm{Al}_{2} \mathrm{O}_{3}$ /agate weight ratio of 2 for 48 hours. Milling was continued for a further 48 hours after the addition of PESf (6.1 wt\%). The suspension was then transferred to a gas tight reservoir and degassed under vacuum until no bubbles could be seen at the surface.

Immediately after degassing, the spinning suspensions were pressurized at a gauge pressure of 4.83 $\mathrm{x} 10^{4}-1.38 \times 10^{5} \mathrm{~Pa}(7-20 \mathrm{psig})$ using nitrogen gas and were extruded through a tube-in-orifice spinneret (outer diameter $3 \mathrm{~mm}$, inner diameter $1.2 \mathrm{~mm}$ ) into tap water with an air-gap of between 0 $\mathrm{mm}$ and $150 \mathrm{~mm}$. The procedure is described in detail elsewhere [19]. De-ionized water was used as the internal coagulant at a flow rate of 0.17-0.62 ml/s. Suspension compositions, viscosities and spinning parameters are given in Table 1.

The fibre precursors were left in the external coagulation bath overnight to allow for completion of phase inversion. They were then immersed in an excess of tap water which was replaced periodically over a period of 48 hours in order to remove traces of NMP. Finally, the fibre precursors were calcined in air (CARBOLITE furnace) to yield ceramic hollow fibre membranes. The temperature was increased from room temperature to $600{ }^{\circ} \mathrm{C}$ at a rate of $2{ }^{\circ} \mathrm{C} / \mathrm{min}$ and held for 2 hours, then to $1000{ }^{\circ} \mathrm{C}$ at a rate of $5{ }^{\circ} \mathrm{C} / \mathrm{min}$ and held for 2 hours and finally to $1450{ }^{\circ} \mathrm{C}$ at a rate of $5{ }^{\circ} \mathrm{C} / \mathrm{min}$ and held for 4 hours. The temperature was then reduced to room temperature at a rate of $5^{\circ} \mathrm{C} / \mathrm{min}$.

\section{Characterization}

Viscosity data was collected (Physica UDS-200 rheometer) using cone and plate geometry at shear rates between 6-100 s ${ }^{-1}$ at $20{ }^{\circ} \mathrm{C}$. Spinning suspension samples were taken and tested immediately prior to fibre spinning. SEM characterization was conducted for both precursor and sintered fibres. The precursor fibres were immersed in liquid nitrogen for 10 minutes after which they were flexed until a clean cross-sectional fracture occurred. Sintered fibres required no such treatment and were flexed at ambient temperature until a fracture occurred. Samples were gold coated under vacuum for 3 minutes at $20 \mathrm{~mA}$ (EMITECH Model K550) and SEM images at varying magnifications were collected (JEOL JSM - 5610 LV). Mercury intrusion data was collected at absolute pressures of between $1.38 \times 10^{3}$ and $2.28 \times 10^{8} \mathrm{~Pa}(0.2$ - 33000 psia) (Micromeritics Autopore IV) with an equilibration time of 10 seconds. The fibres were broken into sections of approximately $4 \mathrm{~mm}$ in length prior to mercury intrusion 
analysis. Gas permeation analysis was performed using nitrogen gas at gauge pressures of between 6.89 $\mathrm{x} 10^{4}$ and $3.45 \times 10^{5} \mathrm{~Pa}(10-50 \mathrm{psig})$ after the fibres were sealed at one end using epoxy resin and fixed into a stainless steel holder to form a test module, also using epoxy resin.

\section{Results and discussion}

Figures 1a-c show precursor fibres spun with air gaps of $0 \mathrm{~mm}, 20 \mathrm{~mm}$ and $150 \mathrm{~mm}$, an internal coagulant flow rate of $0.17 \mathrm{ml} / \mathrm{s}$ and an extrusion velocity of approximately $35 \mathrm{~mm} / \mathrm{s}$. It can be seen from Figure 1a that the morphology of fibres spun directly into a non-solvent bath, i.e. a 0 mm air-gap consists of finger-like voids originating from both the inner and outer fibre surfaces which extend almost to the centre of the fibre cross section. A central sponge-like region is present which provides the majority of the mechanical strength and separation characteristics. Maximum void length is approximately the same for voids originating from both the inner and outer surfaces and as in all the prepared fibres a void length distribution exists, some being only a few microns in length while others penetrate far into the fibre cross-section. This structure may not be ideal for some of the principal applications of ceramic hollow fibre membranes such as solvent filtration which generally require the separation layer (packed pore mostly originated from the sponge-like region after heat treatment) to be at either the inner or outer edge. However, the above structure may be beneficial for the development of catalytic membranes, as finger-like voids may serve as substrates for catalyst particle impregnation. For example, a multifunctional catalytic membrane could be developed with different catalytic functions at the inner and outer surfaces with the central region of the membrane determining the permeation characteristics. This could be achieved by depositing two different types of catalyst targeted at different reactions within the inner and outer finger-like voids, respectively.

Figure 1b shows the fibre morphology resulting from a $20 \mathrm{~mm}$ air-gap. As shown, the finger-like voids extend from the inner surface across approximately $50 \%$ of the fibre cross-section but void length at the outer surface has been greatly reduced. A sponge-like region occupying approximately $35 \%$ of the fibre cross-section is present between the inner and outer finger-like voids. The size and number of voids at the outer edge are further reduced when the air-gap is increased to $150 \mathrm{~mm}$ as shown in Figure 1c. As can be seen, finger-like voids extend from the inner surface across approximately $80 \%$ of the fibre cross-section with the remaining $20 \%$ consisting of a sponge-like region. Close examination of the outer edge of the fibre, as shown in Figure 1d at increased magnification, reveals the presence of 
small finger-like voids despite the increased air-gap. However, it is observed from Figure 1e that these small voids can be eliminated with high temperature treatment.

Previous studies have shown that densification of sponge-like regions occurs during calcination, causing a decrease in porosity, eventually resulting in a gas tight membrane at high calcination temperatures and high $\mathrm{Al}_{2} \mathrm{O}_{3} / \mathrm{PESf}$ ratios [20]. A comparison of Figures 1c and 1f of precursor and calcined fibres spun with an air-gap of $150 \mathrm{~mm}$ suggests that similar results have also been observed in this work and that finger-like voids are retained during calcination despite the densification of the sponge-like structure. This is further demonstrated by a comparison of the mercury intrusion data shown in Figure 2 for fibre $3(150 \mathrm{~mm}$ air-gap, $0.17 \mathrm{ml} / \mathrm{s})$ sintered at $1450{ }^{\circ} \mathrm{C}$ and $1600{ }^{\circ} \mathrm{C}$. For the fibre sintered at $1450{ }^{\circ} \mathrm{C}$ the data shows a bimodal pore size distribution consisting of a peak at approximately $6 \mu \mathrm{m}$ - which represents the pores at the inner fibre surface which form the entrances to the finger-like voids - in addition to a broad peak at approximately $0.16 \mu \mathrm{m}$ which represents the sponge-like region. As the sintering temperature is increased to $1600{ }^{\circ} \mathrm{C}$ the total absence of the peak at $0.16 \mu \mathrm{m}$ indicates that the porosity of the sponge-like region has been totally eliminated. In contrast, the peak for the pores at the inner surface is still present and can be found at approximately $7 \mu \mathrm{m}$, indicating that the porosity in this region and the finger-like void structures remain even at elevated sintering temperature. Gas permeation data for Fibre $3(150 \mathrm{~mm}$ air-gap, $0.17 \mathrm{ml} / \mathrm{s})$, sintered at temperatures of between $1200{ }^{\circ} \mathrm{C}$ and $1600{ }^{\circ} \mathrm{C}$ is shown in Figure 3 and also demonstrates the progressive densification of the sponge-like region of the fibre. As the sintering temperature is increased the gas permeation flux decreases, suffering a dramatic drop between $1400{ }^{\circ} \mathrm{C}$ and $1450{ }^{\circ} \mathrm{C}$. At $1500{ }^{\circ} \mathrm{C}$ the permeation flux is barely detectable and is shown to be totally eliminated in fibres sintered at $1600{ }^{\circ} \mathrm{C}$.

The morphology of the fibre shown in Figure 1a is believed to result from hydrodynamically unstable viscous fingering occurring simultaneously and to a similar extent at both the inner and outer fibre surfaces. The situation is somewhat different when a $20 \mathrm{~mm}$ air-gap is present. In this case simultaneous solvent evaporation and moisture (non-solvent) condensation causes a local viscosity increase in the outer region of the fibre prior to immersion. During the time the nascent fibre is exposed to the atmosphere (20 mm air-gap), the viscosity of the outer region increases and finger-like voids originating at the inner surface penetrate into the fibre cross section. As the nascent fibre makes contact with the non-solvent bath the increased viscosity of the outer region, as a result of exposure to the atmosphere, inhibits the growth of finger-like voids at the outer edge. However, non-solvent influx 
from the precipitation bath does still occur, further increasing the suspension viscosity as it penetrates the nascent fibre cross-section. This increases the viscosity in front of finger-like voids growing from the inner surface, limiting their length to approximately $50 \%$ of the fibre cross-section. Therefore finger-like voids forming from the inner surface can be increased in length by increasing the air-gap to $150 \mathrm{~mm}$ as shown in Figure 1c. Under these conditions the viscosity of the outer region is higher when the nascent fibre is immersed and non-solvent influx is reduced, while at the same time voids originating at the inner surface have more time to penetrate further towards the outer edge. The presence of small finger-like voids in the outer region shows that the suspension viscosity in this region is not above the threshold at which the finger-like voids can be totally suppressed. This is discussed below.

To investigate the effect of viscosity on fibre morphology, water was used as a non-solvent additive to increase the suspension viscosity. Increasing the viscosity by other means, such as by varying the ratio of solvent/polymer/alumina or by the introduction of additives such as polyvinylpyrrolidone (PVP), may affect the membrane properties considerably. For this reason, water was chosen as a viscosity enhancer. The addition of water to spinning suspensions, measured as a percentage of the total solvent content, caused an increase in viscosity as shown in Figure 4. The suspension viscosity value of 12.1 Pa.s at 0 wt\% water increased to 16.7 Pa.s, 18.5 Pa.s and 22 Pa.s at 2 wt\%, 4 wt\% and 6 wt \% water content respectively. Further addition of water resulted in large viscosity increases to 33.3 Pa.s and 45 Pa.s at $8 \mathrm{wt} \%$ and $10 \mathrm{wt} \%$ respectively. The rate of viscosity increase as a function of water content is seen to increase at $6 \mathrm{wt} \%$ indicating that if all other parameters remain the same, fibre morphology may change abruptly at this point. The extrusion pressure was increased in line with viscosity in order to maintain a constant extrusion rate over the range of suspension compositions.

Figure 5 illustrates the effect of spinning suspension viscosity on fibre morphology for calcined fibres prepared with a $150 \mathrm{~mm}$ air-gap and an internal coagulant flow rate of $0.17 \mathrm{ml} / \mathrm{s}$. It can be seen in Figure 5a that at $0 \mathrm{wt} \%$ water content, finger-like voids extent across approximately $80 \%$ of the fibre cross-section with the remaining $20 \%$ consisting of a sponge-like region. At higher magnification, Figure 5b shows the existence of small finger-like voids at the outer fibre edge which are present in fibres prepared with water concentrations below $6 \mathrm{wt} \%$. Addition of $2 \mathrm{wt} \%$ water results in a large reduction in finger-like void length to approximately $40 \%$ of the cross-section as shown in Figure 5c and in the appearance of inward facing isolated voids in the centre of the sponge-like region which can be seen in Figures 5d and 5e. Figure $5 f$ shows the effect of the addition of 4 wt\% water. Again, finger- 
like voids from the inner surface penetrate about $40 \%$ of the cross-section but isolated void formation is reduced in this case. The total elimination of isolated voids resulting from the addition of 6 wt\% water can be seen in Figure $5 g$ and at higher magnification, Figure 5h shows the elimination of small finger-like voids at the outer edge. However, it is shown in Figure $5 g$ that void penetration from the inner surface remained unchanged at $40 \%$ while Figure $5 \mathrm{i}$ shows a reduction in void length to about 30 $\%$ at $8 \mathrm{wt} \%$ water content. Total void elimination resulted with the addition $10 \mathrm{wt} \%$ water, i.e. the symmetric membrane structure shown in Figure 5j.

Figures 6a-6e show fibres prepared with internal coagulant flow rates adjusted so that the ratio of flow rate to viscosity at $30 \mathrm{~s}^{-1}=1.4 \times 10^{-2}$, i.e. the value for fibre $3(0 \mathrm{wt} \%$ water, Figure $5 \mathrm{a})$, thus partially overcoming the resistance to viscous fingering at the inner surface. In this case isolated voids were not observed in any of the fibres prepared and finger-like void length varied in a more linear fashion, i.e. decreasing from $80 \%$ at 0 wt $\%$ water to $70 \%, 60 \%, 40 \%, 35 \%$ and $25 \%$ for water contents of 2 wt\%, $4 \mathrm{wt} \%, 6 \mathrm{wt} \%, 8 \mathrm{wt} \%$ and $10 \mathrm{wt} \%$ respectively. It can be seen at higher magnification that the small finger-like voids at the outer fibre edge observed in Figure $6 \mathrm{f}$ at water concentrations below 6 wt\% were eliminated at 6wt \% water and above as shown in Figure 6g.

The presence of water in the spinning suspension has two effects. Firstly, the initial suspension viscosity is increased and secondly, the suspension viscosity increases more rapidly when in contact with non-solvent, or as a result of solvent evaporation, as the polymer phase is closer to its precipitation point. An increase in suspension viscosity inhibits viscous fingering at both the inner and outer surfaces of the fibre and finger-like void length is reduced. As expected, increasing the internal coagulant flow rate promotes viscous fingering so finger-like void length is reduced less in fibres prepared with increased flow rates. Figures 5c, 5f, 6a and 6b show fibres prepared with 2 wt\% and 4 wt\% water at different internal coagulant flow rates. Close examination of the of the outer fibre edges reveals small finger-like voids - shown at higher magnification in Figures 5b and 6f - resulting from non-solvent influx from the precipitation bath which, although small, are likely to diminish mechanical strength by reducing the integrity of the sponge-like structure. Despite the presence of a $150 \mathrm{~mm}$ air-gap, at water concentrations below $6 \mathrm{wt} \%$ the viscosity of the outer region is below the viscous fingering threshold when the fibre is immersed and small finger-like voids are seen in all fibres regardless of the internal coagulant flow rate. These small finger-like voids are eliminated if the viscosity of the outer region is above a critical value before the outer fibre surface is brought into contact with non-solvent. This threshold is exceeded for fibres prepared with at least $6 \mathrm{wt} \%$ water and a $150 \mathrm{~mm}$ air gap. 
It can also be seen from Figures $5 \mathrm{~d}$ and 5e that the inward facing isolated voids observed in fibres prepared with less than $6 \mathrm{wt} \%$ water content and an internal coagulant flow rate of $0.17 \mathrm{ml} / \mathrm{s}$ are formed by non-solvent influx from the shell side of the fibre. Even if the viscosity near the suspension/air interface has increased considerably before immersion, non-solvent will still diffuse into and through this region when the nascent fibre is immersed. As non-solvent diffuses through the outer sponge-like region of high viscosity and reaches a region of lower viscosity, viscous fingering takes place and isolated void formation is initiated. Isolated voids will be eliminated if the suspension viscosity in this region is above the viscous fingering threshold before non-solvent influx occurs. This is not the case for fibres prepared at $2 \mathrm{wt} \%$ and $4 \mathrm{wt} \%$ water with an internal coagulant flow rate of 0.17 $\mathrm{ml} / \mathrm{s}$. However, Figures 6a-6e show that isolated voids are not observed in any fibres prepared with increased internal coagulant flow rates. Under these circumstances, internal coagulant influx and finger-like void growth from the inner surface are greater. Consequently, the viscosity of the region in which isolated voids are observed is above the viscous fingering threshold before non-solvent influx from the precipitation bath occurs and a sponge-like structure results.

\section{Conclusions}

Finger-like voids in hollow fibres prepared from Alumina/NMP/polyethersulfone spinning suspensions result from hydrodynamically unstable viscous fingering occurring at the interface between the suspension and the non-solvent. Above a critical suspension viscosity this phenomenon is not observed and a sponge-like membrane structure is formed. By varying the viscosity of the spinning suspension by using water as a non-solvent additive, fibre morphology can be varied greatly. Exposure of the outer fibre surface to the atmosphere causes a local viscosity increase and inhibits the formation of fingerlike voids in this region. However, non-solvent may diffuse through this outer layer as the fibre is immersed, halting finger-like void growth from the inner surface and creating isolated voids within the membrane cross-section if the internal coagulant flow rate is insufficient. The air-gap and suspension viscosity are critical in determining both the formation of finger-like voids and the density of the outer sponge-like region. The addition of water as a non-solvent additive to the spinning suspension causes an increase in viscosity, a reduction in finger-like void length and favours the formation of a spongelike structure at the outer fibre surface if an air-gap is present. 


\section{Acknowledgements}

The authors gratefully acknowledge the research funding provided by EPSRC in the United Kingdom (grant No. EP/E000231/1). A project studentship provided by EPSRC to one of the authors, Benjamin F. K. Kingsbury is also gratefully acknowledged. 


\section{References}

[1] C. Guizard, A. Ayral, and A. Julbe, Potentiality of organic solvents filtration with ceramic membranes. A comparison with polymer membranes, Desalination 147 (1-3) (2002) 275-280.

[2] S. Lee and J. Cho, Comparison of ceramic and polymeric membranes for natural organic matter (NOM) removal, Desalination 160 (3) (2004) 223-232.

[3] R. Weber, H. Chmiel, and V. Mavrov, Characteristics and application of new ceramic nanofiltration membranes, Desalination 157 (1-3) (2003) 113-125.

[4] J. Galuszka, R.N. Pandey, and S. Ahmed, Methane conversion to syngas in a palladium membrane reactor, Catalysis Today 46 (2-3) (1998) 83-89.

[5] J.N. Keuler and L. Lorenzen, The dehydrogenation of 2-butanol in a Pd-Ag membrane reactor, J. Membr. Sci. 202 (1-2) (2002) 17-26.

[6] H.W.J.P. Neomagus, G. Saracco, H.F.W. Wessel, and G.F. Versteeg, The catalytic combustion of natural gas in a membrane reactor with separate feed of reactants, Chem. Eng. J. 77 (3) (2000) 165-177.

[7] C.C. Wei and K. Li, Yttria-Stabilized Zirconia (YSZ)-Based Hollow Fiber Solid Oxide Fuel Cells, Ind. Eng. Chem. Res. 47 (5) (2008) 1506-1512.

[8] S. Koonaphapdeelert and K. Li, The development of ceramic hollow fibre membranes for a membrane contactor, Desalination 200 (1-3) (2006) 581-583.

[9] A. Julbe, D. Farrusseng, and C. Guizard, Porous ceramic membranes for catalytic reactors -overview and new ideas, J. Membr. Sci. 181 (1) (2001) 3-20.

[10] S. Loeb and S. Sourirajan, Sea water demineralization by means of an osmotic membrane, Adv. Chem. Ser. 38 (1963) 117-132.

[11] Z.S. Li, Investigation of the dynamics membrane formation by of poly(ether sulfone) membrane formation by precipitation immersion, J. Polym. Sci. Part B: Polym. Phys. 43 (5) (2005) 498-510.

[12] S.A. McKelvey and W.J. Koros, Phase separation, vitrification, and the manifestation of macrovoids in polymeric asymmetric membranes, J. Membr. Sci. 112 (1) (1996) 29-39.

[13] J. Barzin and B. Sadatnia, Theoretical phase diagram calculation and membrane morphology evaluation for water/solvent/polyethersulfone systems, Polymer 48 (6) (2007) 1620-1631.

[14] J. Ren, Z. Li, and F.S. Wong, Membrane structure control of BTDA-TDI/MDI (P84) co-polyimide asymmetric membranes by wet-phase inversion process, J. Membr. Sci. 241 (2) (2004) 305-314.

[15] K.W. Lee, B.K. Seo, S.T. Nam, and M.J. Han, Trade-off between thermodynamic enhancement and kinetic hindrance during phase inversion in the preparation of polysulfone membranes, Desalination 159 (3) (2003) 289-296.

[16] M.R. Pekny, J. Zartman, W.B. Krantz, A.R. Greenberg, and P. Todd, Flow-visualization during macrovoid pore formation in dry-cast cellulose acetate membranes, J. Membr. Sci. 211 (1) (2003) 71-90.

[17] M.A. Frommer and R.M. Messalem, Mechanism of Membrane Formation. VI. Convective Flows and Large Void Formation during Membrane Precipitation, Ind. Eng. Chem. Prod. Res. Dev. 12 (4) (1973) 328-333.

[18] K. Li, X. Tan, and Y. Liu, Single-step fabrication of ceramic hollow fibers for oxygen permeation, J. Membr. Sci. 272 (1-2) (2006) 1-5.

[19] X. Tan, S. Liu, and K. Li, Preparation and characterization of inorganic hollow fiber membranes, J. Membr. Sci. 188 (1) (2001) 87-95.

[20] S. Liu, K. Li, and R. Hughes, Preparation of porous aluminium oxide (Al2O3) hollow fibre membranes by a combined phase-inversion and sintering method, Ceram. Int. 29 (8) (2003) 875881. 
Table 1

$\begin{array}{cccccc}\text { Fibre } & \begin{array}{c}\text { Solvent } \\ \text { composition } \\ \text { (NMP/water) }\end{array} & \begin{array}{c}\text { Bore fluid flow } \\ \text { rate }(\mathbf{m l} / \mathbf{s})\end{array} & \begin{array}{c}\text { Extrusion pressure } \\ \mathbf{( P a )}\end{array} & \begin{array}{c}\text { Air-gap } \\ (\mathbf{m m})\end{array} & \begin{array}{c}\text { Viscosity } \\ @ 30 \mathbf{s}^{-1} \\ \mathbf{( P a . s})\end{array} \\ 1 & 100 / 0 & 0.17 & 7.58 \times 10^{4}(11 \mathrm{psig}) & 0 & 12.1 \\ 2 & 100 / 0 & 0.17 & 7.58 \times 10^{4}(11 \mathrm{psig}) & 20 & 12.1 \\ 3 & 100 / 0 & 0.17 & 7.58 \times 10^{4}(11 \mathrm{psig}) & 150 & 12.1 \\ 4 & 98 / 2 & 0.17 & 7.58 \times 10^{4}(11 \mathrm{psig}) & 150 & 16.7 \\ 5 & 96 / 4 & 0.17 & 1.03 \times 10^{5}(15 \mathrm{psig}) & 150 & 18.5 \\ 6 & 94 / 6 & 0.17 & 1.03 \times 10^{5}(15 \mathrm{psig}) & 150 & 22 \\ 7 & 92 / 8 & 0.17 & 1.03 \times 10^{5}(15 \mathrm{psig}) & 150 & 33.3 \\ 8 & 90 / 10 & 0.17 & 1.38 \times 10^{5}(20 \mathrm{psig}) & 150 & 45 \\ 9 & 98 / 2 & 0.23 & 7.58 \times 10^{4}(11 \mathrm{psig}) & 150 & 16.7 \\ 10 & 96 / 4 & 0.26 & 1.03 \times 10^{5}(15 \mathrm{psig}) & 150 & 18.5 \\ 11 & 94 / 6 & 0.30 & 1.03 \times 10^{5}(15 \mathrm{psig}) & 150 & 22 \\ 12 & 92 / 8 & 0.46 & 1.03 \times 10^{5}(15 \mathrm{psig}) & 150 & 33.3 \\ 13 & 90 / 10 & 0.62 & 1.38 \times 10^{5}(20 \mathrm{psig}) & 150 & 45\end{array}$




\section{Captions for figures}

Figure 1: Cross-sectional images of fibres 1-3: a-c: Precursor fibres, d-f: sintered fibres; (a): Fibre 1, 0 mm air-gap, (b): Fibre 2, 20 mm air-gap and (c): Fibre 3, 150 mm air-gap, (d) Outer edge of fibre 3 (150 mm air-gap) calcined at $1450^{\circ} \mathrm{C}$, (e) Outer edge of fibre 3 (150 mm air-gap) calcined at $1600{ }^{\circ} \mathrm{C}$ and (f): Fibre 3, (150 mm air-gap) calcined at $1450{ }^{\circ} \mathrm{C}$.

Figure 2: Mercury intrusion data for fibre 3 (150 mm air-gap, $0.17 \mathrm{ml} / \mathrm{s}$ ) sintered at $1450{ }^{\circ} \mathrm{C}$ and 1600 ${ }^{\mathrm{o}} \mathrm{C}$.

Figure 3: Gas permeation data for fibre 3 (150 mm air-gap, $0.17 \mathrm{ml} / \mathrm{s}$ ) sintered at temperatures of between $1200{ }^{\circ} \mathrm{C}$ and $1600{ }^{\circ} \mathrm{C}$.

Figure 4: Viscosity of spinning suspensions prepared with $0 \mathrm{wt} \%-10 \mathrm{wt} \%$ water (as a percentage of the total solvent content).

Figure 5: Cross sectional images of fibres 3-8 made from spinning suspensions containing varying amounts of water (a): Fibre 3, 0 wt\% water, (b): Enlargement of Fibre 5 outer edge, 4 wt\% water, (c): Fibre 4, 2 wt\% water, (d): Isolated void in Fibre 5, 4 wt\% water, (e): Enlargement of isolated void in Fibre 5, 4 wt\% water, (f): Fibre 5, 4 wt\% water, (g): Fibre 6, 6 wt\% water, (h): Enlargement of Fibre 6 outer edge, 6 wt $\%$ water, (i): Fibre 7, 8 wt\% water, (j): Fibre 8, $10 \mathrm{wt} \%$ water.

Figure 6: Cross sectional images of fibres 9-13 made from spinning suspensions containing varying amounts of water and spun with different internal coagulant flow rates (a): Fibre 9, 2 wt\% water, $0.23 \mathrm{ml} / \mathrm{s}$, (b): Fibre 10, 4 wt\% water, $0.26 \mathrm{ml} / \mathrm{s}$, (c): Fibre 11, $6 \mathrm{wt} \%$ water, $0.30 \mathrm{ml} / \mathrm{s}$, (d): Fibre 12, 8 wt\% water, 0.46 ml/s, (e): Fibre 13, 10 wt\% water, 0.62 ml/s, (f): Fibre 10, 4 wt\% water, $0.26 \mathrm{ml} / \mathrm{s}$ (outer edge) and (g): Fibre 11, 6 wt\% water, $0.3 \mathrm{ml} / \mathrm{s}$ (outer edge). 
Figure 1

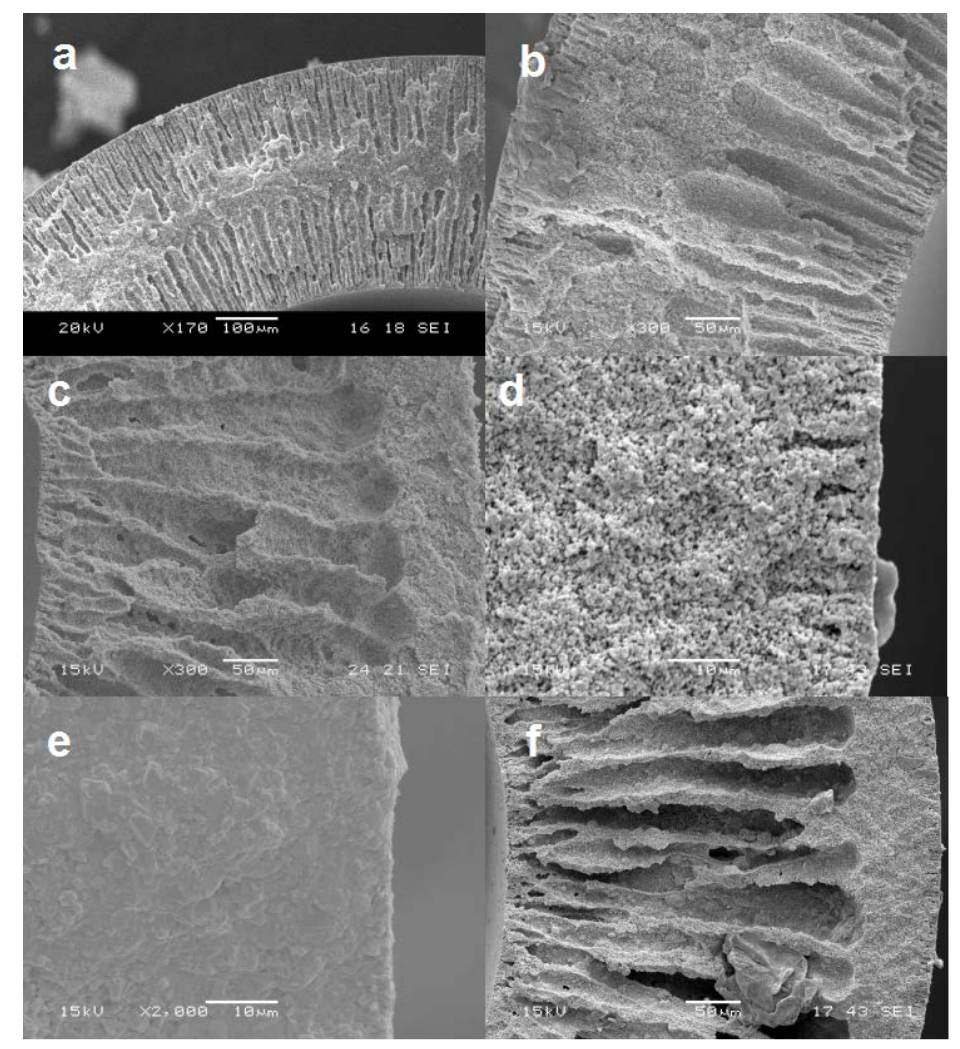


Figure 2

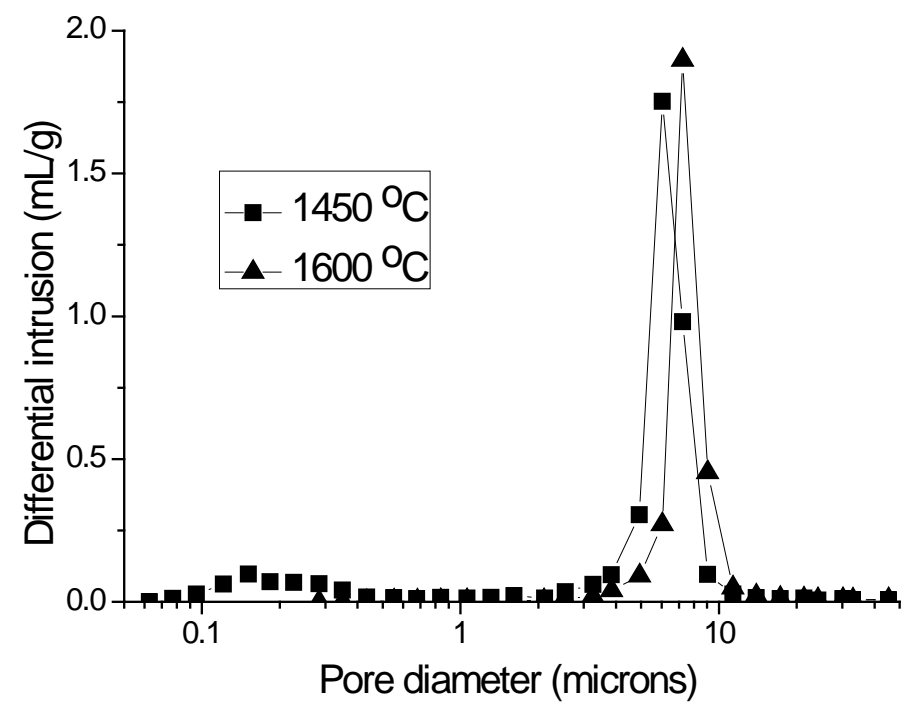


Figure 3

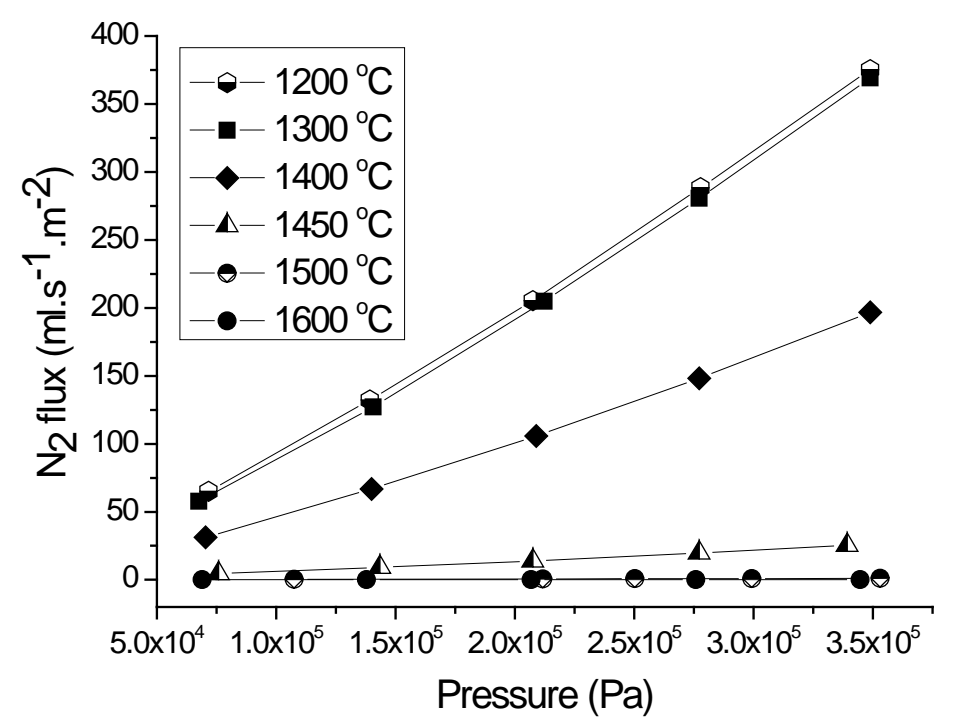


Figure 4

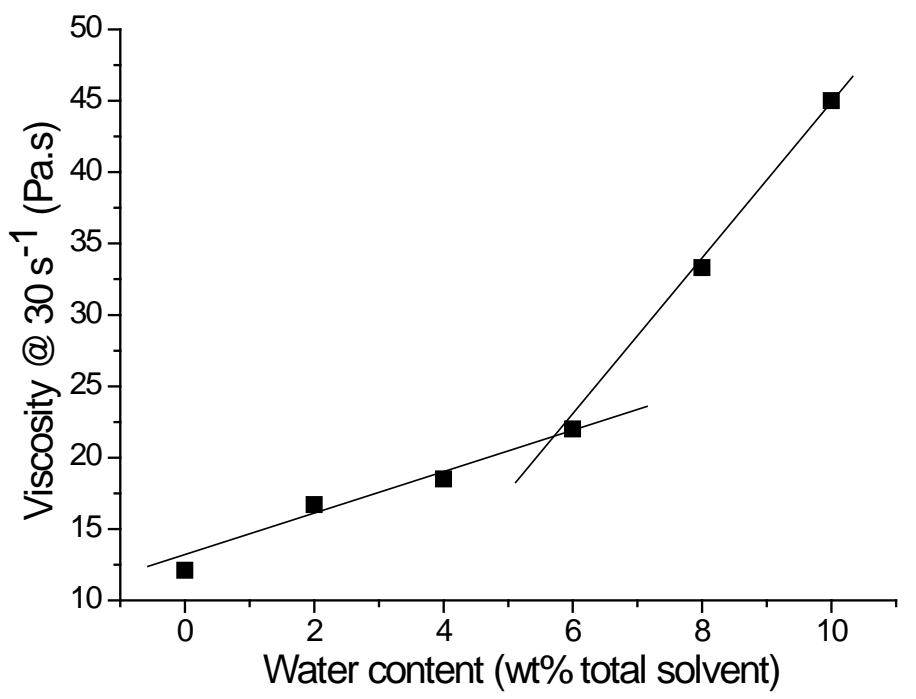


Figure 5

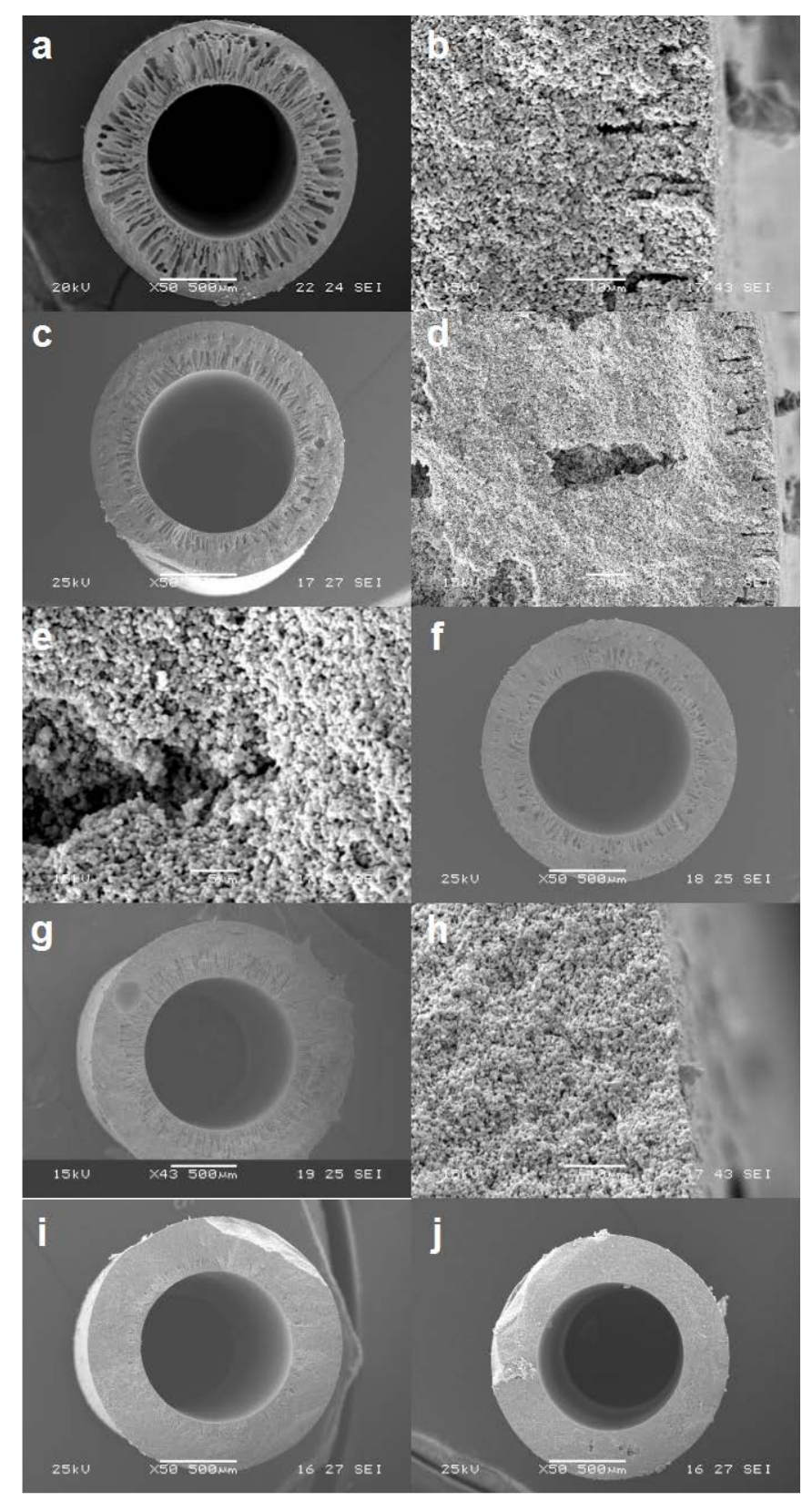


Figure 6

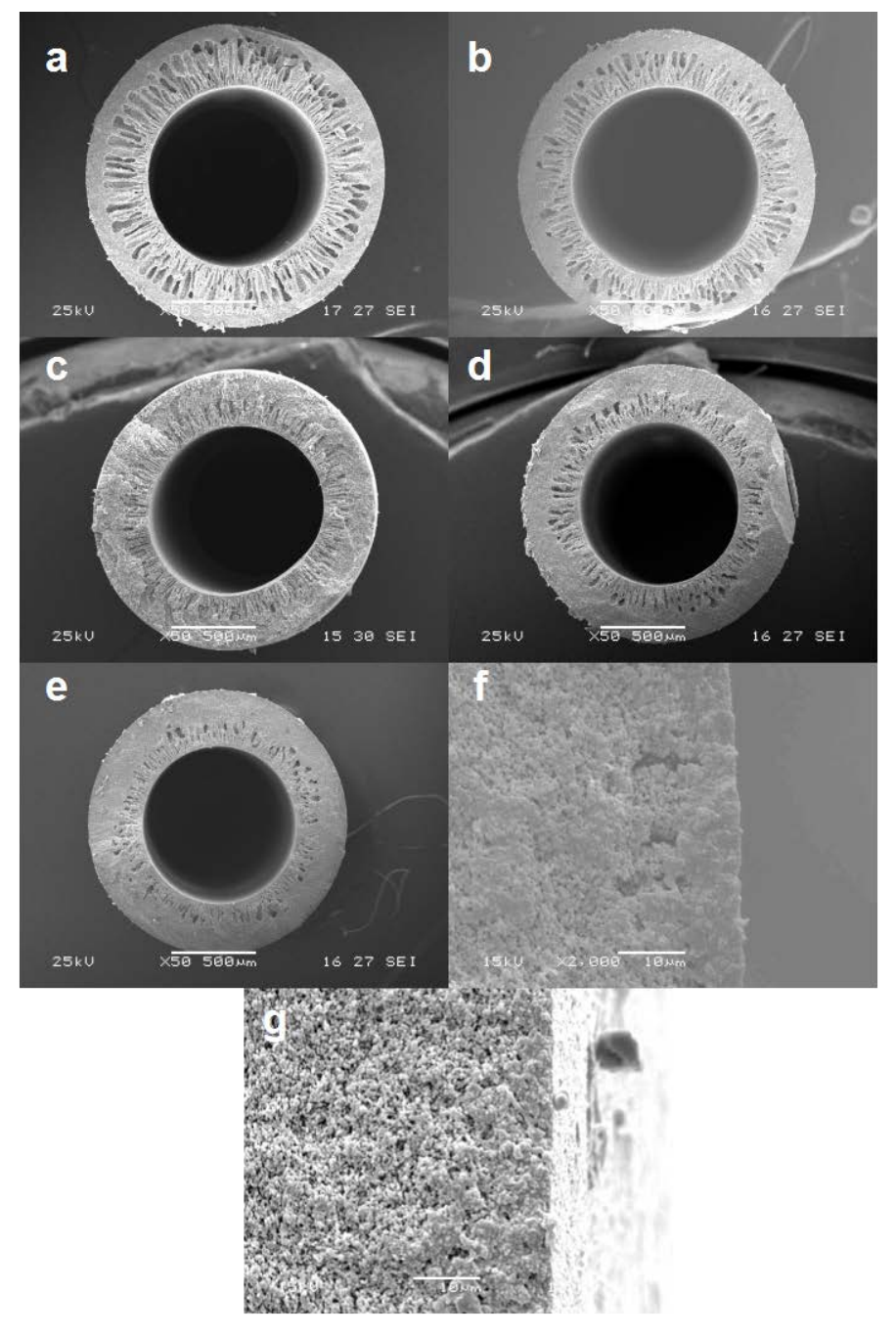

